\title{
Nimotuzumab plus chemotherapy versus chemotherapy alone in advanced non-small- cell lung cancer: a multicenter, randomized, open-label Phase II study
}

\author{
This article was published in the following Dove Press journal: \\ OncoTargets and Therapy \\ 13 June 2014 \\ Number of times this article has been viewed
}

\author{
K Govind Babu' \\ Kumar Prabhash ${ }^{2}$ \\ Ashok K Vaid ${ }^{3}$ \\ Bhawna Sirohi ${ }^{3}$ \\ Ravi B Diwakar ${ }^{4}$ \\ Raghunadha $\mathrm{RaO}^{5}$ \\ Madhuchanda $\mathrm{Kar}^{6}$ \\ Hemant Malhotra ${ }^{7}$ \\ Shona $\mathrm{Nag}^{8}$ \\ Chanchal Goswami ${ }^{9}$ \\ Vinod Raina ${ }^{10}$ \\ Ravi Mohan"I \\ 'Kidwai Memorial Institute of \\ Oncology, Bangalore, ${ }^{2}$ Tata Memorial \\ Hospital, Mumbai, ${ }^{3}$ Artemis Health \\ Institute, Delhi, ${ }^{4}$ Bangalore Institute of \\ Oncology, Bangalore, ${ }^{5}$ Nizam Institute \\ of Medical Sciences, Hyderabad, \\ ${ }^{6} \mathrm{~B}$ R Singh Hospital, Kolkata, ${ }^{7}$ Birla \\ Cancer Centre, Jaipur, ${ }^{8}$ Jehangir \\ Hospital, Pune, 'B P Poddar Hospital \\ and Medical Research Ltd, Kolkata, \\ ${ }^{1}$ Institute Rotary Cancer Hospital, \\ New Delhi, "King George Hospital, \\ Visakhapatnam, India
}

Background: The purpose of this study was to evaluate the safety and efficacy of nimotuzumab in combination with chemotherapy (docetaxel and carboplatin) versus chemotherapy alone in patients with stage IIIB/IV non-small-cell lung cancer.

Methods: This multicenter, open-label, Phase II study randomized 110 patients to receive nimotuzumab plus chemotherapy (nimotuzumab group) or chemotherapy alone (control group), and comprised concomitant, maintenance, and follow-up phases. Nimotuzumab $200 \mathrm{mg}$ was administered once weekly for 13 weeks during the first two phases with four cycles of chemotherapy and docetaxel $75 \mathrm{mg} / \mathrm{m}^{2}$ and carboplatin (area under the curve $5 \mathrm{mg} / \mathrm{mL}^{*} \mathrm{~min}$ ) every 3 weeks for a maximum of four cycles during the concomitant phase. The primary endpoint was objective response rate (sum of complete response and partial response). Secondary endpoints, ie, overall survival and progression-free survival, were estimated using the Kaplan-Meier method. Efficacy was evaluated on the intent-to-treat and efficacy-evaluable sets. Safety was assessed from adverse event and serious adverse event data.

Results: The objective response rate was significantly higher in the nimotuzumab group than in the control group in the intent-to-treat population ( $54 \%$ versus $34.5 \% ; P=0.04)$. A complete response and partial response were achieved in $3.6 \%$ and $50 \%$ of patients, respectively, in the nimotuzumab group, and in $4 \%$ and $30.9 \%$ of patients, respectively, in the control group. No significant differences in median progression-free survival and overall survival were observed. Safety profiles were comparable between the two groups.

Conclusion: Nimotuzumab plus chemotherapy significantly improved the objective response rate as compared with chemotherapy alone. The combination was safe and well tolerated in patients with stage IIIB/IV non-small-cell lung cancer.

Keywords: carboplatin, docetaxel, epidermal growth factor receptor, nimotuzumab, non-small cell lung cancer

\section{Introduction}

Lung cancer is one of the leading causes of cancer-related mortality globally. ${ }^{1}$ Standard methods for the treatment of non-small-cell lung cancer (NSCLC) include surgical resection, radical or palliative radiotherapies, and platinum-based chemotherapies. ${ }^{2}$ The effectiveness of chemotherapy regimens has not improved in recent years, ${ }^{3}$ and has necessitated the development of targeted therapies. The epidermal growth factor receptor (EGFR) is overexpressed in several cancers, ${ }^{4-6}$ including NSCLC. ${ }^{7}$ Since inhibition of EGFR can impede tumor growth, it is a promising candidate for targeted therapy.
Correspondence: Kumar Prabhash

Tata Memorial Hospital, Dr E Borges

Road, Parel, Mumbai - 400 0I2, India

Tel +2224177214

Fax +2224171734

Email kprabhashI@gmail.com 
EGFR is commonly inhibited by tyrosine kinase inhibitors and anti-EGFR monoclonal antibodies. The tyrosine kinase inhibitors gefinitib and erlotinib have been approved by the US Food and Drug Administration (FDA) for the treatment of NSCLC. ${ }^{8,9}$ Unlike tyrosine kinase inhibitors, monoclonal antibodies irreversibly inhibit EGFR by binding to its extracellular domain ${ }^{10}$ and causing receptor internalization, degradation, and long-term downregulation. ${ }^{11,12}$ Further, monoclonal antibodies of the immunoglobulin G1 isotype are capable of recruiting host immune functions, such as antibody-dependent cellular cytotoxicity, in order to attack targeted cancer cells. ${ }^{11}$ The monoclonal antibodies cetuximab and panitumumab are approved by the FDA for use in various cancers, ${ }^{13,14}$ and the former has been extensively studied in NSCLC. ${ }^{11}$ Their main side effect is skin toxicity, and interestingly, this has been associated with greater effectiveness of these drugs. ${ }^{15,16}$

Nimotuzumab is a humanized anti-EGFR mouse monoclonal antibody ${ }^{17}$ designed to reduce immunoreactivity and a slower rate of clearance from the body. ${ }^{18}$ It has been approved in 27 countries for various indications, including pediatric and adult glioma, and head and neck, nasopharyngeal, and esophageal cancers. Clinical trials are also investigating this drug for NSCLC and colorectal, pancreatic, cervical, and breast cancers. ${ }^{18}$ The main advantage of nimotuzumab is that, unlike other EGFR antibodies, it does not cause severe skin toxicity nor does it result in hypomagnesemia or gastrointestinal adverse events. ${ }^{16,19,20}$ Earlier clinical studies of nimotuzumab plus radiation in patients with stage IIB, IIIB, or IV NSCLC have demonstrated that this combination therapy was well tolerated and feasible for patients unsuitable for radical therapy. ${ }^{21,22}$ Nimotuzumab combined with gemcitabine and cisplatin was also safe and tolerable in patients with advanced NSCLC. ${ }^{23}$ Additional studies evaluating the safety and efficacy of nimotuzumab in combination with various chemotherapeutic agents are ongoing. ${ }^{18}$

The objective of the present study was to assess the safety and efficacy of nimotuzumab in combination with chemotherapy consisting of docetaxel and carboplatin as compared with chemotherapy alone in the treatment of patients with stage IIIB/IV NSCLC. The primary endpoint was the objective response rate. Secondary endpoints included assessment of overall survival and progression-free survival, and the safety and tolerability of this combination.

\section{Materials and methods Study design}

This multicenter, randomized, open-label, active-controlled, prospective Phase II study was designed to evaluate the safety and efficacy of nimotuzumab (BIOMAb-EGFR) in combination with chemotherapy (docetaxel and carboplatin) in the treatment of patients with stage IIIB/IV NSCLC. Patients were randomly assigned to receive nimotuzumab plus chemotherapy (nimotuzumab group) or chemotherapy alone (control group), based on a centerwise, 1:1, permutated block randomization scheme. The study comprised concomitant, maintenance, and follow-up phases, and was conducted at eleven centers across India (Figure 1). Patients were moved to the next stage only if they demonstrated an objective response (complete response/partial response/ stable disease) according to the Response Evaluation Criteria in Solid Tumors (RECIST) version 1.1 at the end of each stage. ${ }^{24}$ Patients showing progressive disease at any stage were discontinued from the study drug but were followed up for survival until the end of the trial. When disease progression was recorded at any stage of the trial, further treatment was administered at the investigators' discretion. The participants or observers were not blinded because the study endpoints were objective parameters and bias was considered minimal. Ethical approval was obtained from their respective institutional ethical review boards. The study was conducted according to the ethical principles laid down in the Declaration of Helsinki, and was consistent with the ethical guidelines for Biomedical Research on Human Subjects issued by the Indian Council of Medical Research and the guidelines for Clinical Trials on Pharmaceutical Products

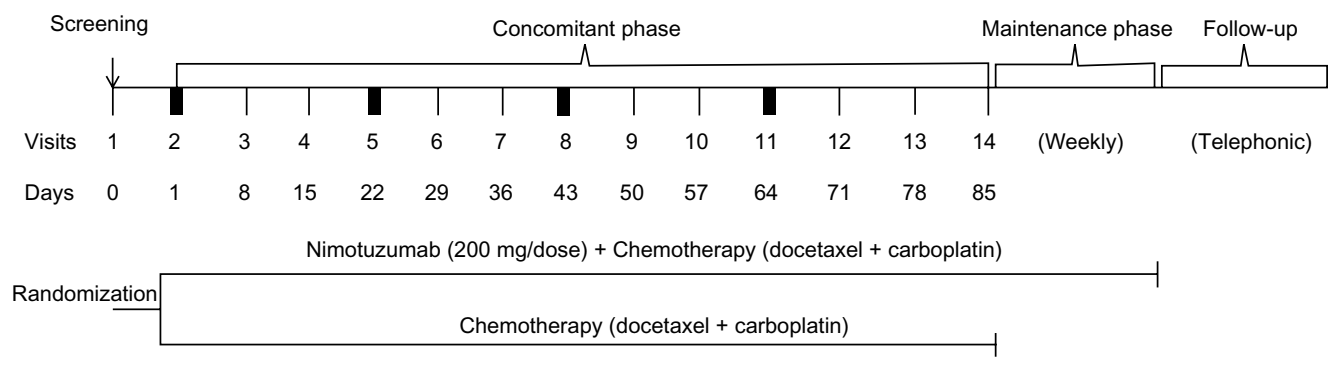

Figure I Study design. 
in India-Good Clinical Practice Guidelines, issued by the Central Drugs Standard Control Organization, Ministry of Health, Government of India.

\section{Participants}

Indian patients of either sex and aged $\geq 18$ years who had unresectable stage IIIB/IV NSCLC with at least one lesion that had not been irradiated previously and could be measured by RECIST, who were treatment naïve, and met all inclusion criteria were enrolled in the study. Detailed inclusion and exclusion criteria are provided as supplementary material. All patients provided their written informed consent.

\section{Intervention}

Nimotuzumab $200 \mathrm{mg}$ in $250 \mathrm{~mL}$ saline was administered intravenously over 30 minutes once weekly, and docetaxel $75 \mathrm{mg} / \mathrm{m}^{2}$ was administered intravenously over one hour followed by carboplatin over 30 minutes every 3 weeks. The $200 \mathrm{mg}$ dose of nimotuzumab was selected because this dose is reported to be as effective as $400 \mathrm{mg} .^{22}$ The dose of docetaxel was adjusted to $65 \mathrm{mg} / \mathrm{m}^{2}$ for patients whose nadir platelet count during previous therapy was $<25,000$ cells $/ \mathrm{mm}^{3}$, those who experienced febrile neutropenia, and those who had grade 3 or higher nonhematologic toxicities. In patients who required further dose reduction, a dose of $50 \mathrm{mg} / \mathrm{m}^{2}$ was administered. The carboplatin dosage was determined by the Calvert formula based on the patient's pre-existing renal function. During the concomitant phase, patients in the nimotuzumab group received nimotuzumab weekly for 13 weeks along with chemotherapy, whereas the control group received chemotherapy alone. In both groups, chemotherapy was administered once every 3 weeks for a maximum of four cycles or until the development of progressive disease or unacceptable toxicity. In the maintenance phase, patients in the nimotuzumab group continued to receive the study drug weekly until development of progressive disease or the end of the study. No active treatment was administered in the control group. In the follow-up phase, patients showing disease progression during the concomitant or maintenance phase were contacted by telephone every 2 months for assessment of survival.

\section{Assessments}

The primary endpoint was objective response rate, defined as the sum of complete response and partial response, and was a direct measure of the drug's antitumor activity. Response was assessed using RECIST. ${ }^{24}$ Secondary endpoints included overall survival, progression-free survival, and the safety and tolerability profiles of each regimen. Safety was assessed based on the incidence of adverse events, serious adverse events, laboratory parameters, electrocardiograms, vital signs, and clinical parameters.

\section{Statistical analysis}

The objective response rate was calculated as the proportion of patients showing a complete response or partial response during the study. The Kaplan-Meier method was used to estimate the $95 \%$ confidence intervals (CIs) of overall survival and progression-free survival. Overall survival was calculated from the date of randomization to the date of death from any cause. Progression-free survival was defined as the time from the start of treatment to the time of disease progression or the last scheduled visit. Survival distributions of both groups were compared using a log-rank test. The mean and standard error for overall survival and progression-free survival were also recorded. Continuous variables were summarized using mean, standard deviation, median, and range (minimum and maximum), while categorical variables were summarized using proportions (counts and percentages). The tests were two-sided, and were considered significant at $P \leq 0.05$. In total, 110 patients were to be recruited to obtain 100 evaluable patients ( 50 in each study group), considering a dropout rate of $10 \%$. Sample size for this study was not calculated statistically. Efficacy was evaluated from two population sets, ie, the intent-to-treat set including all patients who were exposed to at least one dose of the study drug and returned for at least one visit after initiation of treatment, and the efficacy-evaluable set including patients who received at least two cycles of chemotherapy and had one assessment of tumor response. Subgroup analysis was conducted based on prognostic criteria.

\section{Results}

A total of 134 patients were screened; 110 were randomized and 24 were screening failures. The study was completed by three $(5.7 \%)$ and four $(7.0 \%)$ patients in the nimotuzumab and control groups, respectively, without disease progression (Figure 2). Demographic and baseline characteristics were similar between the two groups (Table 1). Of the 110 patients, 109 were included in the intent-to-treat population, ie, $53(100 \%)$ and $56(98.2 \%)$ in the nimotuzumab and control groups, respectively. Further, 94 of the 110 patients were included in the efficacy-evaluable population, ie, 45 (84.9\%) and $49(86 \%)$ in the nimotuzumab and control groups, respectively. 


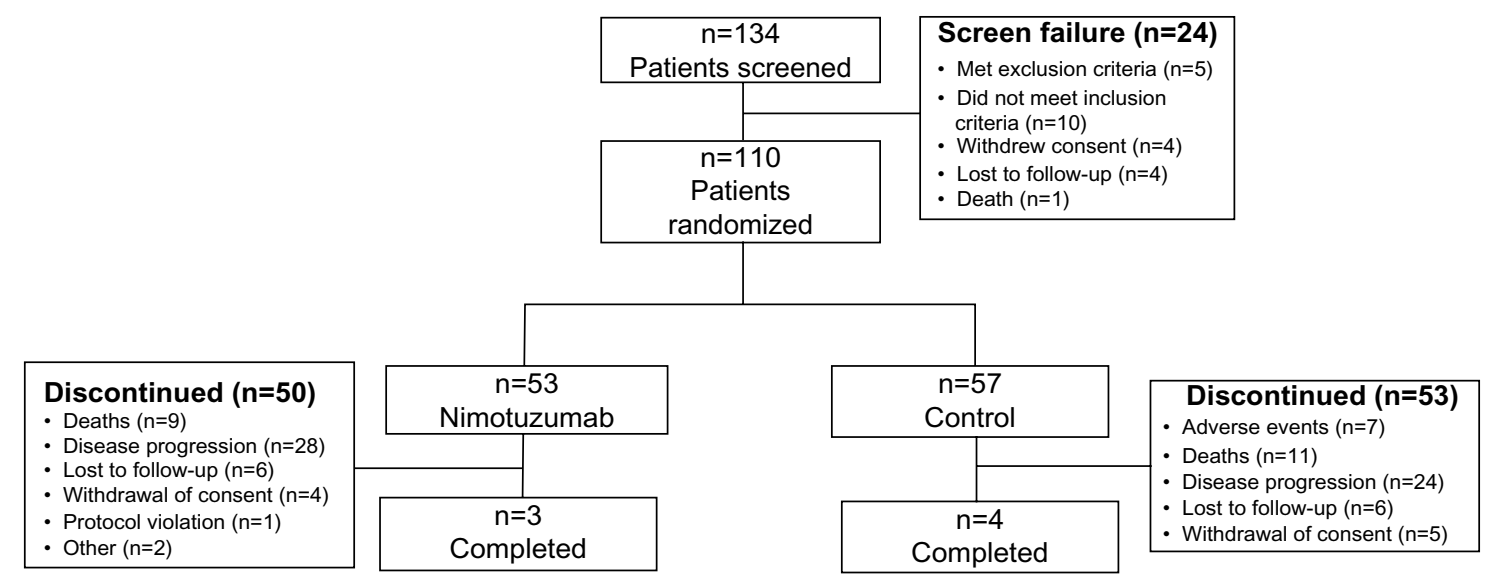

Figure 2 Patient disposition.

\section{Efficacy}

\section{Primary endpoint}

The primary efficacy endpoint was the objective response rate. In the intent-to-treat population, a complete response was achieved in two patients each in the nimotuzumab (4\%) and control (3.6\%) groups, while a partial response was achieved in $25(50 \%)$ and $17(30.9 \%)$ of patients in the nimotuzumab and control groups, respectively (Figure 3). Statistically significant differences in objective response rate were observed between the nimotuzumab and control groups $(54 \%, 95 \%$ CI $40.2-67.8$ versus $34.5 \%, 95 \%$

Table I Patient demographics and baseline characteristics

\begin{tabular}{|c|c|c|}
\hline & $\begin{array}{l}\text { Nimotuzumab } \\
(n=53)\end{array}$ & $\begin{array}{l}\text { Control } \\
(n=57)\end{array}$ \\
\hline \multicolumn{3}{|l|}{ Demographic characteristics } \\
\hline \multicolumn{3}{|l|}{ Sex, n (\%) } \\
\hline Male & $4 \mathrm{I}(77.4)$ & $45(78.9)$ \\
\hline Female & $12(22.6)$ & $12(2||)$. \\
\hline Mean age (SD), years & $58.2(9.5)$ & $55.6(10.7)$ \\
\hline Mean weight (SD), kg & $54.3(9.9)$ & $55.1(10.9)$ \\
\hline Mean height (SD), $\mathrm{cm}$ & $161.7(8.9)$ & $161.7(9.7)$ \\
\hline \multicolumn{3}{|l|}{ Baseline characteristics } \\
\hline \multicolumn{3}{|l|}{ ECOG performance status, n (\%) } \\
\hline 1 & $40(75.5)$ & $47(82.5)$ \\
\hline 0 & $13(24.5)$ & $10(17.5)$ \\
\hline Mean time to diagnosis (SD), days & $20.6(30.7)$ & $24.7(35.4)$ \\
\hline \multicolumn{3}{|l|}{ Stage of disease, $\mathrm{n}(\%)$} \\
\hline IIIB & $25(47.2)$ & $22(38.6)$ \\
\hline IV & $28(52.8)$ & $35(61.4)$ \\
\hline \multicolumn{3}{|l|}{ Histopathology, n (\%) } \\
\hline Adenocarcinoma & $29(54.72)$ & $40(70.18)$ \\
\hline Large cell carcinoma & I (I.89) & $2(3.5 I)$ \\
\hline Squamous cell carcinoma & $15(28.30)$ & $9(15.80)$ \\
\hline Undifferentiated and others & $8(15.09)$ & $6(10.53)$ \\
\hline $\begin{array}{l}\text { Mean sum of longest diameters } \\
\text { for target lesion }(S D)\end{array}$ & $125.3(6 \mathrm{I})$ & I I $0.8(58.8)$ \\
\hline
\end{tabular}

Abbreviations: ECOG, Eastern Cooperative Oncology Group; SD, standard deviation.
CI $22-47.1 ; P=0.04)$. The trend in the efficacy-evaluable population was similar, with a significant difference in objective response rate between the nimotuzumab and control groups (60\%, 95\% CI 45.7-74.3 versus $38.8 \%$, 95\% CI 25.1-52.4; $P=0.04)$. Subgroup analyses for the objective response rate were conducted by including known prognostic variables such as sex, tobacco use, stage of NSCLC, Eastern Cooperative Oncology Group performance status, histopathology, and age. In the intent-to-treat population, the objective response rate was higher in the nimotuzumab group than in the control group for all subgroups, with the exception of the subgroup for undifferentiated histopathology (Table 2). However, no subgroup, except for the non-tobacco user group $(P=0.01)$, showed a statistically significant difference. Similar results were observed in the efficacy-evaluable population.

\section{Secondary endpoints}

The secondary efficacy endpoints included overall survival and progression-free survival, which were evaluated during a median follow-up period of 26.17 months. In the intentto-treat population, the difference in median overall survival was not statistically significant between the nimotuzumab and control groups (10.1 months, 95\% CI 8.1-13.6 versus 10.4 months, $95 \%$ CI $7.4-13.7 ; P=0.48)$. The hazard ratio was 0.844 (95\% CI 0.529-1.35) in the nimotuzumab group relative to the control group (Figure 4). A similar trend was noted in the efficacy-evaluable population. The median overall survival was 10.1 months $(95 \%$ CI $8.8-13.6)$ in the nimotuzumab group versus 12.8 months (95\% CI 8.8-15.9) in the control group $(P=0.66)$. The hazard ratio for the nimotuzumab group was $0.892(95 \%$ CI $0.537-1.482)$ relative to the control group. Like the overall survival results, the difference in progression-free survival in the intent-to-treat population was not statistically significant $(P=0.31)$ between 

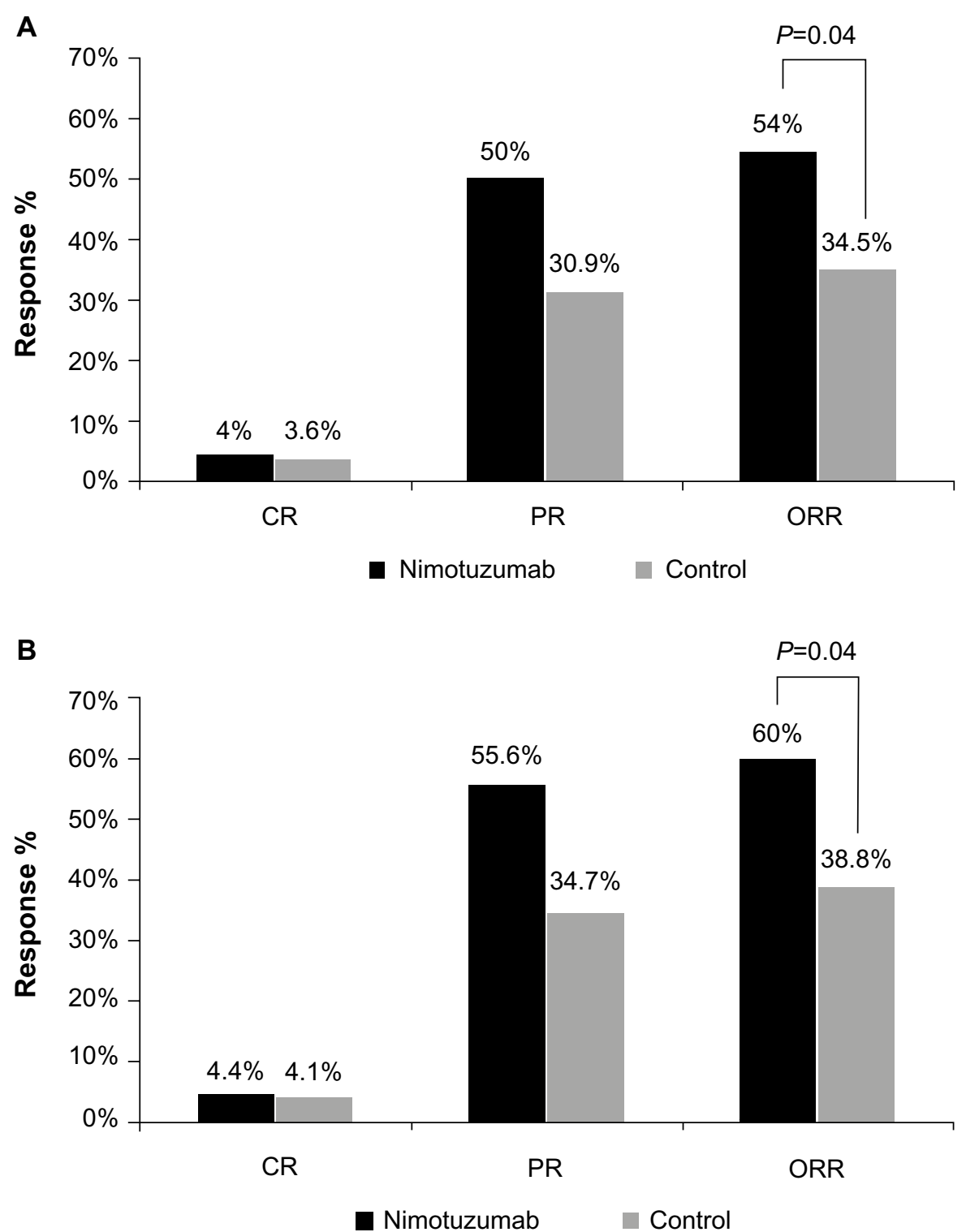

Figure 3 ORRs of the (A) intent-to-treat and (B) efficacy-evaluable populations.

Notes: Patients with non-small-cell lung cancer were treated with nimotuzumab plus chemotherapy (nimotuzumab) or chemotherapy alone (control). The ORR was measured during tumor evaluation visits and is the sum of the $C R$ and $P R$.

Abbreviations: $C R$, complete response; $P R$, partial response; $O R R$, objective response rate.

the nimotuzumab (4.9 months, 95\% CI 4.5-6.9) and control (4.8 months, 95\% CI 2.8-5.8) groups. The hazard ratio was 0.807 (95\% CI 0.533-1.222) for the nimotuzumab group relative to the control group. There was a similar trend in the efficacy-evaluable population, with a median progressionfree survival of 6.5 months $(95 \%$ CI $4.6-8.3)$ in the nimotuzumab group and 5 months $(95 \%$ CI 4.6-6.1) in the control group $(P=0.54)$.

\section{Safety}

All patients who received at least one dose of the study drug were included in the safety analysis. The median cumulative dose and duration of exposure to the study drug was 3,800 (range 600-10,400) $\mathrm{mg}$ and 135 (range 15-359) days, respectively, up to the end of the study. A total of 781 adverse events were reported during the study, of which 402 occurred in the nimotuzumab group and 379 in the control group (Table 3). At least one adverse event was reported by $43(81.1 \%)$ and $47(82.5 \%)$ patients in the nimotuzumab and control groups, respectively. In the nimotuzumab arm, the most commonly reported adverse events overall were decreased appetite (28.3\%; 15 patients) and cough $(24.5 \% ; 13$ patients). The most common nimotuzumab-related adverse events were vomiting $(5.7 \%$; three patients), diarrhea (3.8\%; two patients), fatigue (3.8\%; two patients), pain (3.8\%; two patients) 
Table 2 Objective response rate with subgroup analysis (ITT population)

\begin{tabular}{|c|c|c|c|}
\hline Variable & Arm & $\begin{array}{l}\text { ORR (number of ORs; } \\
\text { percent of OR [ } 95 \% \\
\text { Cl for percent]) }\end{array}$ & $P$-value \\
\hline \multirow[t]{2}{*}{ Male sex } & Control arm $(n=43)$ & $13 ; 30.2(16.5-44.0)$ & 0.0522 \\
\hline & BIOMAb arm $(n=39)$ & $20 ; 51.3(35.6-67.0)$ & \\
\hline \multirow[t]{2}{*}{ Female sex } & Control arm $(n=12)$ & $6 ; 50.0(21.7-78.3)$ & 0.5099 \\
\hline & BIOMAb arm $(n=I I)$ & $7 ; 63.6(35.2-92.1)$ & \\
\hline \multirow[t]{2}{*}{ Tobacco user } & Control arm $(n=33)$ & I3; $39.4(22.7-56.1)$ & 0.5426 \\
\hline & BIOMAb arm $(n=32)$ & I5; $46.9(29.6-64.2)$ & \\
\hline \multirow[t]{2}{*}{ Non-tobacco user } & Control arm (n=22) & $6 ; 27.3(8.7-45.9)$ & 0.0127 \\
\hline & $\mathrm{BIOMAb}$ arm $(\mathrm{n}=18)$ & I2; 66.7 (44.9-88.4) & \\
\hline \multirow[t]{2}{*}{ Stage IIIB } & Control arm ( $\mathrm{n}=2 \mathrm{I})$ & $7 ; 33.3(13.2-53.5)$ & 0.1606 \\
\hline & BIOMAb arm $(n=24)$ & I3; $54.2(34.2-74.1)$ & \\
\hline \multirow[t]{2}{*}{ Stage IV } & Control arm $(n=34)$ & $12 ; 35.3(|9.2-5| .4)$ & 0.1507 \\
\hline & BIOMAb arm $(n=26)$ & I4; 53.8 (34.7-73.0) & \\
\hline \multirow[t]{2}{*}{ ECOG performance score 0} & Control arm $(n=10)$ & $4 ; 40.0(9.6-70.4)$ & 0.1610 \\
\hline & BIOMAb arm $(n=13)$ & $9 ; 69.2(44.1-94.3)$ & \\
\hline \multirow[t]{2}{*}{ ECOG performance score I } & Control arm $(n=45)$ & $15 ; 33.3(19.6-47.1)$ & 0.1593 \\
\hline & BIOMAb arm $(n=37)$ & I8; $48.6(32.5-64.8)$ & \\
\hline \multirow[t]{2}{*}{ Histopathology adenocarcinoma } & Control arm $(n=39)$ & $14 ; 35.9(20.8-5 \mid .0)$ & 0.0846 \\
\hline & BIOMAb arm $(n=28)$ & I6; $57.1(38.8-75.5)$ & \\
\hline \multirow[t]{2}{*}{ Histopathology large cell carcinoma } & Control arm $(n=0)$ & 0 & 0.0833 \\
\hline & BIOMAb arm $(n=1)$ & I; $100.0(100.0-100.0)$ & \\
\hline \multirow[t]{2}{*}{ Histopathology squamous cell carcinoma } & Control arm $(n=9)$ & $3 ; 33.3(2.5-64.1)$ & 0.1470 \\
\hline & BIOMAb arm $(n=14)$ & $9 ; 64.3(39.2-89.4)$ & \\
\hline \multirow[t]{2}{*}{ Histopathology undifferentiated and others } & Control arm $(n=5)$ & $2 ; 40.0(0.0-82.9)$ & 0.3105 \\
\hline & BIOMAb arm $(n=7)$ & I; I $4.3(0.0-40.2)$ & \\
\hline \multirow[t]{2}{*}{ Age $\leq 65$ years } & Control arm $(n=48)$ & 18; $37.5(23.8-5 \mid .2)$ & 0.1117 \\
\hline & BIOMAb arm $(n=44)$ & $24 ; 54.5(39.8-69.3)$ & \\
\hline \multirow[t]{2}{*}{ Age $>65$ years } & Control arm $(n=7)$ & I; I $4.3(0.0-40.2)$ & 0.1596 \\
\hline & BIOMAb arm $(n=6)$ & $3 ; 50.0(10.0-90.0)$ & \\
\hline
\end{tabular}

Notes: Best objective response among complete response, partial response, stable disease, and progressive disease from start of treatment to end of follow-up is considered. If a patient's objective response is not available (due to, eg, withdrawal or loss to follow-up) then it was not considered in either denominator or numerator for calculating percentage. $P$-value calculated using two-sample proportion test.

Abbreviations: $\mathrm{Cl}$, confidence interval; ITT, intent-to-treat; ECOG, Eastern Cooperative Oncology Group; BIOMAb, nimotuzumab; OR, overall response; ORR, overall response rate.

and rash (3.8\%; two patients). The majority of events in the nimotuzumab group were mild in severity $(66.17 \%$; 266 events). Life-threatening events were reported in eleven $(20.8 \%)$ patients, and events that led to an outcome of death were reported in four $(7.5 \%)$ patients. Of the 62 serious adverse events reported, two (febrile neutropenia and gastroenteritis) were considered related to the study drug. At least one mild adverse event was reported by $15(28.3 \%)$ and $26(45.6 \%)$ patients in the nimotuzumab and control groups, respectively. Laboratory parameters were similar between the nimotuzumab and control groups.

\section{Discussion}

This Phase II trial showed that nimotuzumab was safe and well tolerated when used together with chemotherapy comprising docetaxel and carboplatin in patients with stage IIIB/IV NSCLC. Furthermore, the primary efficacy variable, objective response rate, was significantly higher in the nimotuzumab group (54\%, 95\% CI 40.2-67.8) than in the control group $(34.5 \%, 95 \%$ CI 22-47.1). The objective response rate was also higher for most subgroups by prognostic criteria, although the differences were not statistically significant. No statistically significant between-group difference in overall survival or progression-free survival was observed. The incidence of adverse events was comparable between the groups. Decreased appetite and cough were the most common adverse events in the nimotuzumab group; also reported were vomiting, diarrhea, fatigue, pain, and rash, and each of these was seen in $\leq 6 \%$ of the nimotuzumab-treated patients. These results indicate a better safety profile for nimotuzumab compared with other monoclonal antibodies, given that previous studies of monoclonal antibodies such 


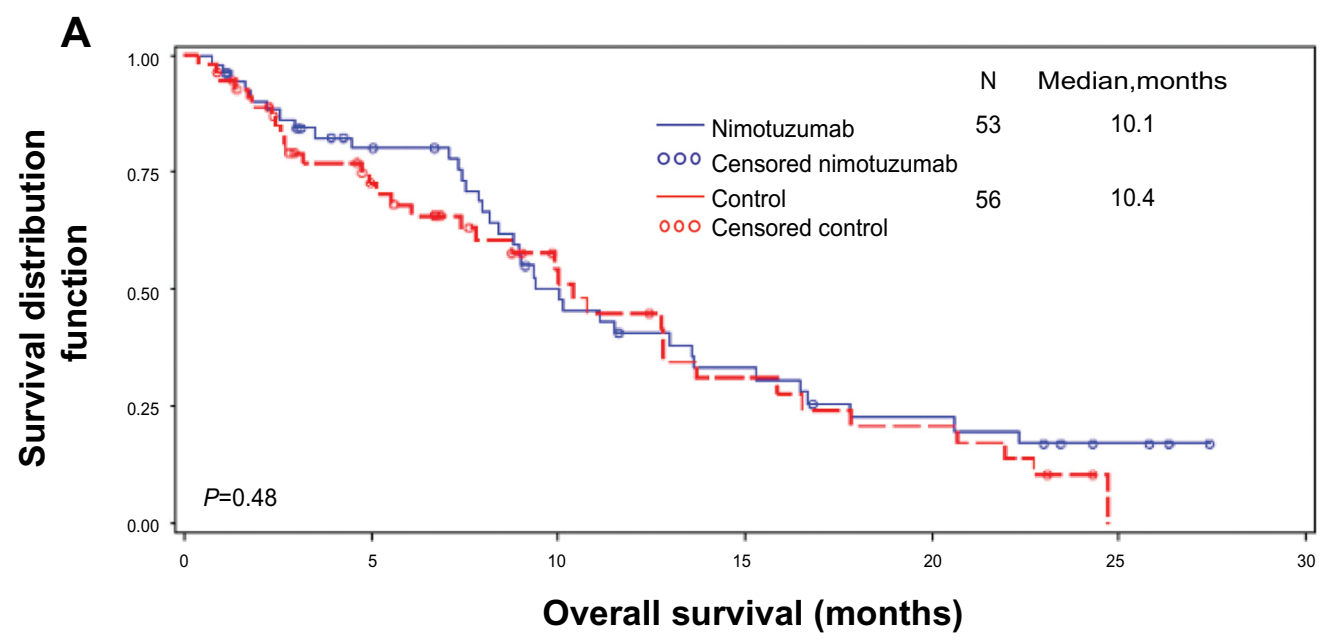

B

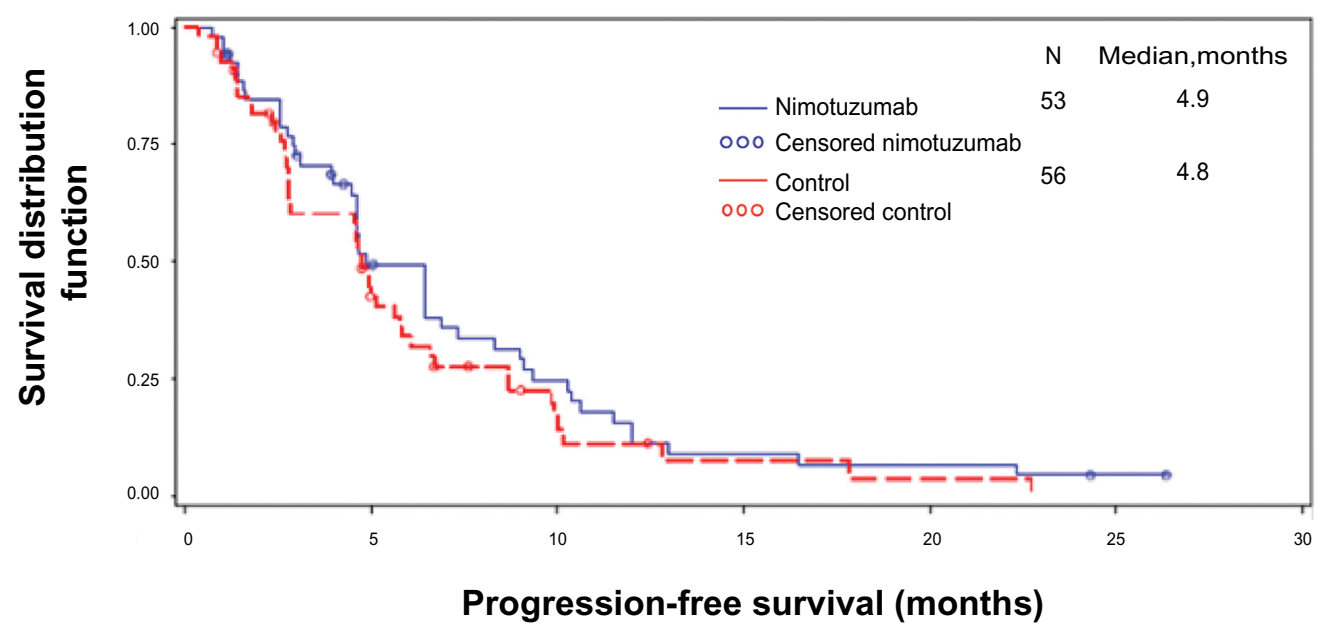

Figure 4 (A) Overall survival and (B) progression-free survival in the intent-to-treat population.

Notes: Overall survival and progression-free survival were estimated during the median follow-up period of 26.17 months. The Kaplan-Meier method was used to estimate median overall survival and progression-free survival of patients with non-small-cell lung cancer in the nimotuzumab (blue) or control (red) groups. The survival distribution of the two treatment groups was compared using a log-rank test.

as cetuximab, matuzumab, and panitumumab have demonstrated a higher $(60 \%-80 \%)$ incidence of skin toxicities. ${ }^{15}$

The objective response rate achieved in our study (54\%) is higher than that reported in some previous studies conducted in NSCLC patients administered a combination of EGFR monoclonal antibodies and chemotherapy. A study evaluating the efficacy of nimotuzumab plus gemcitabine and cisplatin as second-line therapy in patients with advanced NSCLC reported a response rate of $25 \% .^{23}$ In the Phase III FLEX (First-Line ErbituX in lung cancer) trial, treatment with cetuximab combined with cisplatin and vinorelbine resulted in a $36 \%$ overall response rate (measured by World Health Organization criteria) compared with $29 \%$ achieved with chemotherapy alone. ${ }^{25}$ Similarly, in the Bristol-Myers Squibb 099 study, conducted in support of the FLEX trial, the overall response rate (measured by World Health Organization criteria) was $25.7 \%$ when cetuximab was administered in combination with a taxane (paclitaxel or docetaxel) and carboplatin. ${ }^{26}$ In addition, a study examining the efficacy of carboplatin and docetaxel in NSCLC has reported an overall response rate of $27.1 \%,{ }^{27}$ which is comparable with the objective response rate achieved with the same chemotherapy combination in our study (29\%).

Overall survival in our study did not show significant between-group differences (hazard ratio 0.844, 95\% CI $0.529-1.346 ; P=0.48)$ in the nimotuzumab group (10.1 months in both the intent-to-treat and efficacy-evaluable populations) relative to the control group (10.4 months and 12.8 months in the intent-to-treat and efficacy-evaluable populations, respectively); however, overall survival in both groups was marginally better than that in some previous studies examining EGFR monoclonal antibodies and various chemotherapeutic agents. The docetaxel and carboplatin combination when administered to patients with stage IIIB/IV 
Table 3 Summary of adverse events

\begin{tabular}{|c|c|c|}
\hline Adverse events & $\begin{array}{l}\text { Nimotuzumab } \\
(n=53)\end{array}$ & $\begin{array}{l}\text { Control } \\
(n=57)\end{array}$ \\
\hline Total & 402 & 379 \\
\hline Patients with at least one $A E, n(\%)$ & $43(81.1)$ & $47(82.5)$ \\
\hline Patients with at least one severe & $24(45.3)$ & $28(49.1)$ \\
\hline \multicolumn{3}{|l|}{$\mathrm{AE}, \mathrm{n}(\%)$} \\
\hline Patients at least one mild AE, n (\%) & I5 (28.3) & $26(45.6)$ \\
\hline \multicolumn{3}{|c|}{$\begin{array}{l}\text { Most common AEs (occurring in more than four patients in any group), } \\
\mathrm{nl} ; \mathrm{n} 2(\%)\end{array}$} \\
\hline Anemia & $12 ; 7(13.2)$ & $6 ; 5(8.8)$ \\
\hline Febrile neutropenia & $3 ; 3(5.7)$ & $5 ; 5(8.8)$ \\
\hline Leukopenia & $12 ; 6(11.3)$ & $9 ; 4(7.0)$ \\
\hline Neutropenia & $15 ; 9(17.0)$ & $10 ; 7(12.3)$ \\
\hline Constipation & $6 ; 5(9.4)$ & $3 ; 3(5.3)$ \\
\hline Diarrhea & $14 ; 9(17.0)$ & $26 ; 16(28.1)$ \\
\hline Nausea & $8 ; 5(9.4)$ & $11 ; 9(15.8)$ \\
\hline Vomiting & $10 ; 9(17.0)$ & $16 ; 14(24.6)$ \\
\hline Asthenia & $15 ; 10(18.9)$ & $13 ; 9(15.8)$ \\
\hline Chest pain & $23 ; 10(18.9)$ & $20 ; 11(19.3)$ \\
\hline Mucosal inflammation & $4 ; 4(7.5)$ & $6 ; 5(8.8)$ \\
\hline Edema peripheral & $13 ; 8(15.1)$ & $2 ; 2(3.5)$ \\
\hline Pain & $16 ; 8(15.1)$ & $13 ; 7(12.3)$ \\
\hline Pyrexia & $22 ; 11(20.8)$ & $22 ; 15(26.3)$ \\
\hline Gastroenteritis & $2 ; 2(3.8)$ & $7 ; 6(10.5)$ \\
\hline Decreased appetite & $34 ; 15(28.3)$ & $24 ; 12(21.1)$ \\
\hline Back pain & $7 ; 4(7.5)$ & $7 ; 7$ (I2.3) \\
\hline Cough & $38 ; 13(24.5)$ & $26 ; 11(19.3)$ \\
\hline Dyspnea & 19; $12(22.6)$ & $27 ; 17$ (29.8) \\
\hline Oropharyngeal pain & $5 ; 5(9.4)$ & $0(0.0)$ \\
\hline Rash & $5 ; 5(9.4)$ & $2 ; 2(3.5)$ \\
\hline
\end{tabular}

Abbreviations: $A E$, adverse event; $n 1$, number of events; $n 2$, number of patients.

NSCLC resulted in an overall survival of 9.2 months $^{27}$ and 9.4 month $^{28}$ in two separate studies. Nimotuzumab in combination with gemcitabine and cisplatin as second-line therapy offered a median overall survival of 9.8 months. ${ }^{23}$ In our study, like overall survival, there were no significant between-group differences in progression-free survival $(P=0.31)$; the median progression-free survival of 4.9 months achieved in the nimotuzumab group was comparable with that of 4.44 months observed in another study using cetuximab in combination with a taxane. ${ }^{26}$

Based on our study results, nimotuzumab seems to produce a higher response rate than other monoclonal antibodies, but does not significantly change overall survival. Previous studies have reported that various factors, such as genetic mutations and nonoptimal administration of EGFR antagonists, may influence the direct effects of EGFR-targeted therapies on overall survival. ${ }^{19,29}$ Perhaps future studies examining the impact of nimotuzumab on survival in greater detail are warranted. Previous clinical trials have already demonstrated the safety and tolerability of nimotuzumab in NSCLC in combination with radiotherapy ${ }^{21,22}$ and a different chemotherapy regimen. ${ }^{23}$ Our results demonstrate that nimotuzumab in combination with docetaxel and carboplatin is safe. In addition, consistent with earlier studies, the incidence of skin rash was low, presenting in only $3.8 \%$ of patients in the nimotuzumab group. The advantage of nimotuzumab over other anti-EGFR antibodies is its benign side effect profile. ${ }^{16}$ This can be attributed to its bivalent binding to the EGFR $^{30}$ and possibly to the fact that it does not disrupt the basal level of EGFR signalling. ${ }^{31}$

The low toxicity and high response rate associated with nimotuzumab suggest that it could be a promising monoclonal antibody for patients with advanced NSCLC. The standard first-line treatment for patients with advanced NSCLC is chemotherapy consisting of a platinum analog combined with either vinorelbine, gemcitabine, pemetrexed (in patients with nonsquamous disease), or a taxane, such as paclitaxel or docetaxel. ${ }^{32}$ Maintenance therapy is administered to patients whose tumor growth has been controlled after a specified number of chemotherapy cycles. This therapy is continued until the occurrence of unacceptable toxicity or disease progression, and requires agents causing minimal toxicity. ${ }^{32}$ While nimotuzumab fits into this definition, further optimization to improve overall survival is warranted.

This study has some limitations. First, the sample size was not calculated statistically. Second, the participants and the observers were not blinded. Although observer bias was considered minimal because the endpoints were objective parameters, a potential for bias might exist. Third, the response of patients to the study drug was evaluated by the investigator but was not confirmed by a radiologist, and this could have led to some bias in the results. Fourth, this is not a histopathologically directed study and randomization was not stratified according to histopathological type. It is possible that a study of nimotuzumab in a histopathologically directed trial would obtain a different result. Finally, it is possible that the second-line treatment may have exerted an effect on overall survival, and this was not taken into account. In conclusion, this study demonstrates that nimotuzumab in combination with chemotherapy comprising docetaxel and carboplatin improved the objective response rate significantly as compared with chemotherapy alone, and the combination was well tolerated without any meaningful safety concerns in patients with stage IIIB/IV NSCLC.

\section{Acknowledgments}

This clinical trial was supported by Biocon Ltd. We acknowledge the contribution made by the coinvestigators 
and Clinigene International Ltd, Bangalore. The authors retained full control of the manuscript content.

\section{Disclosure}

This paper was presented at the 2013 American Society of Clinical Oncology annual meeting and the abstract is included in the proceedings of that meeting. The authors report no other conflicts of interest in this work.

\section{References}

1. Jemal A, Bray F, Center MM, Ferlay J, Ward E, Forman D. Global cancer statistics. CA Cancer J Clin. 2011;61:69-90.

2. Boland $\mathrm{W}, \mathrm{Bebb} \mathrm{G}$. The emerging role of nimotuzumab in the treatment of non-small cell lung cancer. Biologics. 2010;4:289-298.

3. Schiller JH, Harrington D, Belani CP, et al. Comparison of four chemotherapy regimens for advanced non-small-cell lung cancer N Engl J Med. 2002;346:92-98.

4. Slamon DJ, Clark GM, Wong SG, Levin WJ, Ullrich A, McGuire WL. Human breast cancer: correlation of relapse and survival with amplification of the HER-2/neu oncogene. Science. 1987;235:177-182.

5. Hanawa M, Suzuki S, Dobashi Y, et al. EGFR protein overexpression and gene amplification in squamous cell carcinomas of the esophagus. Int J Cancer. 2006;118:1173-1180.

6. Schlomm T, Kirstein P, Iwers L, et al. Clinical significance of epidermal growth factor receptor protein overexpression and gene copy number gains in prostate cancer. Clin Cancer Res. 2007;13:6579-6584.

7. Hirsch FR, Varella-Garcia M, Bunn P Jr, et al. Epidermal growth factor receptor in non-small-cell lung carcinomas: correlation between gene copy number and protein expression and impact on prognosis. $J$ Clin Oncol. 2003;21:3798-3807.

8. Lynch TJ, Bell DW, Sordella R, et al. Activating mutations in the epidermal growth factor receptor underlying responsiveness of non-smallcell lung cancer to gefitinib. $N$ Engl J Med. 2004;350:2129-2139.

9. Díaz A, Lage A. Therapies based on inhibitors of the epidermal growth factor receptor: reaching for the future. Biotecnol Apl. 2007;24:10-18.

10. Pirker R, Filipits M. Monoclonal antibodies against EGFR in non-small cell lung cancer. Crit Rev Oncol Hematol. 2011;80:1-9.

11. Gerber DE. EGFR inhibition in the treatment of non-small cell lung cancer. Drug Dev Res. 2008;69:359-372.

12. Prewett M, Rockwell P, Rockwell RF, et al. The biologic effects of C225, a chimeric monoclonal antibody to the EGFR, on human prostate carcinoma. J Immunother Emphasis Tumor Immunol. 1996;19: 419-427.

13. Pazdur R. FDA approval for cetuximab: National Cancer Institute. Available from: http://www.cancer.gov/cancertopics/druginfo/fdacetuximab. Accessed October 24, 2012.

14. Pazdur R. FDA Approval for panitumumab: National Cancer Institute. Available from: http://www.cancer.gov/cancertopics/druginfo/fdapanitumumab. Accessed October 24, 2012.

15. Agero AL, Dusza SW, Benvenuto-Andrade C, Busam KJ, Myskowski P, Halpern AC. Dermatologic side effects associated with the epidermal growth factor receptor inhibitors. J Am Acad Dermatol. 2006;55: 657-670.

16. Boland WK, Bebb G. Nimotuzumab: a novel anti-EGFR monoclonal antibody that retains anti-EGFR activity while minimizing skin toxicity. Expert Opin Biol Ther. 2009;9:1199-1206.

17. Mateo C, Moreno E, Amour K, Lombardero J, Harris W, Pérez R. Humanization of a mouse monoclonal antibody that blocks the epidermal growth factor receptor: recovery of antagonistic activity. Immunotechnology. 1997;3:71-81.
18. Takeda M, Okamoto I, Nishimura Y, Nagakawa K. Nimotuzumab, a novel monoclonal antibody to the epidermal growth factor receptor, in the treatment of non-small cell lung cancer. Lung Cancer Targets Ther. 2011;2:59-67.

19. Crombet T, Osorio M, Cruz T, et al. Use of the humanized antiepidermal growth factor receptor monoclonal antibody h-R3 in combination with radiotherapy in the treatment of locally advanced head and neck cancer patients. J Clin Oncol. 2004;22:1646-1654.

20. Rodriguez MO, Rivero TC, del Castillo Bahi R, et al. Nimotuzumab plus radiotherapy for unresectable squamous-cell carcinoma of the head and neck. Cancer Biol Ther. 2010;9:343-349.

21. Choi HJ, Sohn JH, Lee CG, et al. A phase I study of nimotuzumab in combination with radiotherapy in stages IIB-IV non-small cell lung cancer unsuitable for radical therapy: Korean results. Lung Cancer. 2011;71:55-59.

22. Bebb G, Smith C, Rorke S, et al. Phase I clinical trial of the antiEGFR monoclonal antibody nimotuzumab with concurrent external thoracic radiotherapy in Canadian patients diagnosed with stage IIb, III or IV non-small cell lung cancer unsuitable for radical therapy. Cancer Chemother Pharmacol. 2011;67:837-845.

23. Wang HQ, Ren Y, Qian ZZ, et al. Nimotuzumab combined with gemcitabine and cisplatin as second-line chemotherapy for advanced nonsmall-cell lung cancer. Thoracic Cancer. 2012;3:72-78. Available from: http://onlinelibrary.wiley.com/doi/10.1111/j.1759-7714.2011.00083.x/ abstract. Accessed May 30, 2014.

24. Therasse P, Arbuck SG, Eisenhauer EA, et al. New guidelines to evaluate the response to treatment in solid tumors. European Organization for Research and Treatment of Cancer, National Cancer Institute of the United States, National Cancer Institute of Canada. J Natl Cancer Inst. 2000;92:205-216.

25. Pirker R, Pereira JR, Szczesna A, et al. Cetuximab plus chemotherapy in patients with advanced non-small-cell lung cancer (FLEX): an open-label randomised phase III trial. Lancet. 2009;373:1525-1531.

26. Lynch TJ, Patel T, Dreisbach L, et al. Cetuximab and first-line taxane/ carboplatin chemotherapy in advanced non-small-cell lung cancer: results of the randomized multicenter phase III trial BMS099. J Clin Oncol. 2010;28:911-917.

27. Socinski MA, Raju RN, Stinchcombe T, et al. Randomized, phase II trial of pemetrexed and carboplatin with or without enzastaurin versus docetaxel and carboplatin as first-line treatment of patients with stage IIIB/IV non-small cell lung cancer. $J$ Thorac Oncol. 2010;5: 1963-1969.

28. Fossella F, Pereira JR, von Pawel J, et al. Randomized, multinational, phase III study of docetaxel plus platinum combinations versus vinorelbine plus cisplatin for advanced non-small-cell lung cancer: the TAX 326 study group. J Clin Oncol. 2003;21:3016-3024.

29. Perez R, Moreno E, Garrido G, Crombet T. EGFR-targeting as a biological therapy: understanding nimotuzumab's clinical effects. Cancer. 2011;3:2014-2031.

30. Garrido G, Tikhomirov IA, Rabasa A, et al. Bivalent binding by intermediate affinity of nimotuzumab: a contribution to explain antibody clinical profile. Cancer Biol Ther. 2011;11:373-382.

31. Talavera A, Friemann R, Gomez-Puerta S, et al. Nimotuzumab, an antitumor antibody that targets the epidermal growth factor receptor, blocks ligand binding while permitting the active receptor conformation. Cancer Res. 2009;69:5851-5859.

32. Thatcher N, Heighway J. Maintenance and consolidation therapy in patients with unresectable stage III/IV non-small cell lung cancer Oncologist. 2010;15:1034-1042. 


\section{Supplementary information Inclusion criteria}

1. Subjects who have a histologically or cytologically confirmed unresectable stage IIIB/IV non-small-cell lung cancer.

2. At least one evaluable lesion which is not irradiated, that can be measured using Response Evaluation Criteria in Solid Tumors.

3. Subjects who are treatment-naïve.

4. Should have completed at least 4 weeks from last major surgery and prior radiation therapy to the pelvis, spine, or long bones and recovered from side effects.

5. Subjects must have Eastern Cooperative Oncology Group performance status of 0 to 1 .

6. Subjects of either sex aged $\geq 18$ years.

7. Subjects with life expectancy $\geq 12$ weeks.

8. Subjects who are willing to use effective methods of contraception starting with visit 1 and for at least 30 days after the last dose of study medication.

9. Laboratory parameters within normal ranges that are defined for this study by:

- hematology (hemoglobin $\geq 10 \mathrm{~g} / \mathrm{L}$, absolute neutrophil count $\geq 1,500$ cells $/ \mathrm{mm}^{3}$, platelets $\geq 100,000 / \mathrm{mm}^{3}$ )

- liver function (serum total bilirubin $\leq 1.5$ times upper limit of normal [ULN]; aspartate aminotransferase and alanine aminotransferase $\leq 2.5$ times ULN $[\leq 5$ times ULN in the presence of liver metastases]; GGT $\leq 1.5$ times ULN; alkaline phosphatase $\leq 5.0$ times ULN; prothrombin time $\leq 1.5$ times ULN unless receiving therapeutic anticoagulation; partial thromboplastin time $\leq 1.2$ times ULN, unless receiving therapeutic anticoagulation)

- kidney function (serum creatinine $\leq 1.5$ times ULN.
10. Willingness to sign informed consent form by the subject or legally acceptable representative.

\section{Exclusion criteria}

1. Prior chemotherapy or immunotherapy or therapy with another investigational agent.

2. Peripheral neuropathy grade $\geq 2$ (Common Terminology Criteria for Adverse Events version 3.0).

3. Active metastatic central nervous system disease.

4. Prior severe infusion reaction to antibody therapy.

5. Serious uncontrolled medical disorders that would impair the ability to receive therapy.

6. Evidence of QT prolongation of $>440 \mathrm{msec}$ as per the screening electrocardiogram.

7. Subject has a history of or concurrent malignancy except for inactive non-melanoma skin cancer and/or in situ carcinoma of the cervix, or other solid tumour treated curatively.

8. Subject has positive history of human immunodeficiency virus, hepatitis $\mathrm{B}$, and/or hepatitis $\mathrm{C}$ infection.

9. Women who are pregnant or breastfeeding.

10. Altered mental status or psychiatric condition that prohibits understanding or providing consent.

11. Patients with severe underlying disease not controlled by treatment in the opinion of the principal investigator.
OncoTargets and Therapy

\section{Publish your work in this journal}

OncoTargets and Therapy is an international, peer-reviewed, open access journal focusing on the pathological basis of all cancers, potential targets for therapy and treatment protocols employed to improve the management of cancer patients. The journal also focuses on the impact of management programs and new therapeutic agents and protocols on

\section{Dovepress}

patient perspectives such as quality of life, adherence and satisfaction. The manuscript management system is completely online and includes a very quick and fair peer-review system, which is all easy to use. Visit http://www.dovepress.com/testimonials.php to read real quotes from published authors. 